\title{
CORONARY ARTERY DISEASE
}

\section{Calcium density reduces CVD risk}

The Agatston score for coronary artery calcium (CAC) is frequently used to determine the risk of cardiovascular disease (CVD). In new research published in JAMA and presented at the AHA Scientific Sessions, however, calcium density is inversely correlated with the risk of coronary heart disease (CHD) and CVD, suggesting that the upward weighting for calcium density in the Agatston score could result in the misclassification of some patients.

Investigators in this multicentre, multiethnic, prospective, observational study enrolled 3,398 individuals aged 45-84 years who were free from known CVD, but had a CAC score $>0$. After a median of 7.6 years of follow-up, the natural logarithm of the CAC volume score was associated with incident $\mathrm{CHD}$ (HR 1.81 per SD increase, 95\% CI 1.47-2.23). Conversely, the CAC density was inversely associated with incident CHD (HR 0.73 per SD, 95\% CI 0.58-0.91).

Adding the CAC density score to a model used to predict the risk of CHD and CVD significantly improved the model. The area under the curve for the base model that used the Framingham risk score, ethnicity, and statin use was improved from 0.668 to 0.700 when the CAC volume score was added, and to 0.711 when the CAC density score was also added. These data suggest that the Agatston score could be improved by altering the contribution of CAC density to the final score for CAC.

In previous work, patients with stable CHD have been found to have denser plaques than patients with acute heart disease. Similarly, patients treated with statins tend to have a greater increase in CAC density than those not treated with statins, again suggesting that stable plaques have dense calcium deposits.

\section{Megan Cully}

Original article Criqui, M. H. et al. Calcium density of coronary artery plaque and risk of incident cardiovascular events. JAMA doi:10.1001/jama.2013.282535 